\title{
Passivity-based Object-Level Impedance Control for a Multifingered Hand
}

\author{
Thomas Wimboeck, Christian Ott, Gerhard Hirzinger \\ Institute of Robotics and Mechatronics \\ German Aerospace Center (DLR) \\ Oberpfaffenhofen, 82234 Wessling, Germany \\ Email: thomas.wimboeck@dlr.de
}

\begin{abstract}
Holding an object and manipulating it in 6D is a key application for multifingered robot hands. In the past many algorithms were proposed based on a weighted pseudoinverse of the grasp map combined with an internal force control. The majority of these algorithms require robust contact detection/tracking and switching controllers. Employing the virtual object introduced by Stramigioli we present an object-level control law. We define a novel virtual object frame based on the robot hand configuration. Our control law takes a desired object frame and desired grasping forces as input, it is passive, has an intuitive physical meaning, and stability is even given in case a finger looses contact with the object. A damping design as a function of the desired object stiffness and the combined hand-object inertia is presented. The performance of the controller is proven in two experiments implemented on the DLR Hand II.
\end{abstract}

\section{INTRODUCTION}

The research in dexterous manipulation with multifingered hands has been addressed by many researchers over the last decades. A very good overview up to the millenium can be found in [1].

Different controllers have been developed and implemented on the DLR Hand II (Fig. 1) on joint-, Cartesian- and objectlevel [2], [3]. Object-level control has in general the advantages that it is easy to define grasp forces, to compensate for robot and object inertia, to specify external forces acting on the object and to avoid unnecessary high internal forces [4]. The goal of this work was to develop a robust and intuitive control law for object-level manipulation which does not require robust contact detection/tracking. On object-level we command directly object motion without explicitly controlling the tool center points (TCP) of the single fingers or the joint angles. Other requirements for the controller are intrinsic passivity, stability in the case of a finger loosing contact during manipulation, an intuitive physical interpretation, and a damping design w.r.t the desired object-level stiffness and the hand and object inertias.

In [5] Arimoto et al. presented a control law to simultaneously command grasping forces and object orientation for manipulation by a pair of fingers in the plane. Introducing the concept of stability on a manifold the control law was proven to be stable. However, the proposed control law applies only to the manipulation of polygonal $2 \mathrm{D}$ objects in a plane with fingers being equipped with hemispherical tips exploiting

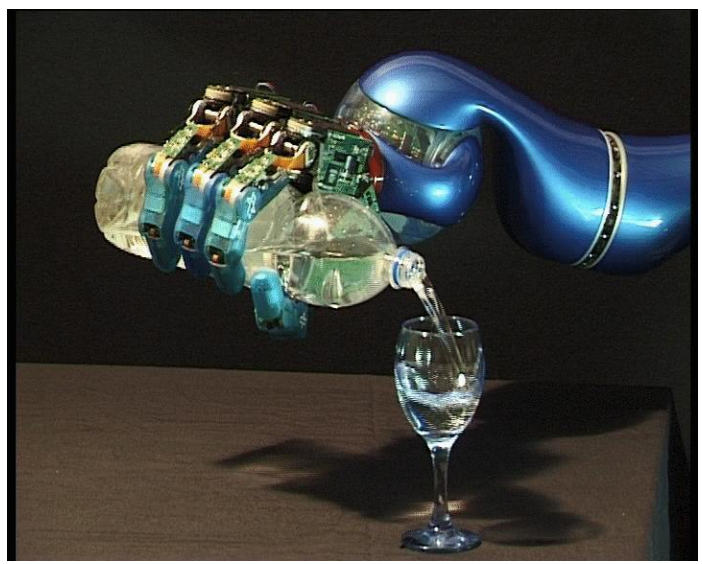

Fig. 1. DLR Hand II mounted on the light-weight arm LBR III serving a glass of water.

rolling constraints.

Williams and Khatib proposed the virtual linkage to construct a basis of the nullspace of the grasp map, respectively for internal forces [6]. Combined with the augmented object model, a control structure for constrained motion, active force control operations and internal force control for rigid grasps was shown in [7].

Schneider and Cannon presented an object-level impedance control law including internal force control [4]. They showed promising experiments for planar manipulations and applied this to an insertion task. The control is based on the weighted pseudoinverse of the grasp map assuming rigid grasps.

Caccavale et al. proposed a joint-level control law in combination with a kinetostatic filter in order to avoid undesired internal forces resulting from the proportional joint error [8]. The kinetostatic filter is based on a weighted pseudoinverse of the grasp map and an additional internal-force controller is added. The different physical meanings of forces and torques require that a certain metric for obtaining an inversion of the grasp map should be used accordingly to [9]. Naniwa and Wada extended the work in [8] with a posture control and studied the control law in experiments with two 3 degrees of freedom (DOF) fingers performing 2D object manipulation [10]. 
Yun and Kumar derive a control law for object motion and internal forces in a plane based on feedback-linearization [11].

Wen and Kreutz-Delgado [12] present control laws on joint-level, Cartesian-level, and for generalized coordinates (e. g. object pose, TCP forces) for multiple-arm systems assuming rigid grasps.

For object manipulation in general the pseudoinverse of the grasp matrix is widely used as proposed in [13]. Bonitz and Hsia use the better suited weighted pseudoinverse [14]. Both cases require the knowledge of the position of the object either from an external sensor (e. g. stereo camera) or from using the net force acting on the object to track its pose using a precise object model.

A different approach is taken by Stramigioli [15] who introduced the concept of a virtual object. Instead of considering actual contact points or detecting contact transitions for force control the fingertips are connected via spatial springs with the virtual object which is additionally connected via the so-called hand configuration spring with a virtual position of the hand. The object pose is determined by considering the net forces and applying these to the virtual object.

Stramigioli used only damping related to the hand configuration spring and not for the fingertip-object connecting springs. If the object is not moving, due to the undamped connecting springs an oscillatory internal motion may occur. We propose to overcome these undesired oscillations by employing dampers parallel to the connecting springs. For passivity reasons we are not shaping the object inertia. Therefore, we need no simulation of the virtual object dynamics.

In this work we propose to define the object pose completely based on the hand configuration. Based on this pose a passivity-based control design is employed yielding to an intrinsically passive control (IPC) to realize object motions and grasp force.

\section{SYSTEM MODEL}

\section{A. Robot Model}

The equations of motion of a robot hand with $N$ fingers and $M=3$ DOF per finger are well known [13]:

$$
\mathbf{M}(\boldsymbol{\theta}) \ddot{\boldsymbol{\theta}}+\mathbf{C}(\boldsymbol{\theta}, \dot{\boldsymbol{\theta}}) \dot{\boldsymbol{\theta}}+\mathbf{g}(\boldsymbol{\theta})=\boldsymbol{\tau}+\boldsymbol{\tau}_{\text {ext }},
$$

$\boldsymbol{\theta}, \boldsymbol{\tau}, \boldsymbol{\tau}_{\text {ext }} \in \mathbb{R}^{3 N}$ are the joint angles, the joint torques and the external torque, respectively. $\mathbf{M}(\boldsymbol{\theta}) \in \mathbb{R}^{3 N \times 3 N}$, $\mathbf{C}(\boldsymbol{\theta}, \dot{\boldsymbol{\theta}}) \dot{\boldsymbol{\theta}}, \mathbf{g}(\boldsymbol{\theta}) \in \mathbb{R}^{3 N}$ represent the joint-level inertia matrix, the centripetal and Coriolis vector and the gravity vector in stacked notation. tions:

In the following derivations we make the following assump-

- The contacts of the fingertips with the object behave like point contacts with friction (PCWF).

- The internal forces provided by the controller are chosen to be sufficient such that the friction constraints are fulfilled for all contact points.

- In order to allow $6 \mathrm{D}$ object motion the contact between the object and the hand are restricted to the fingertips.

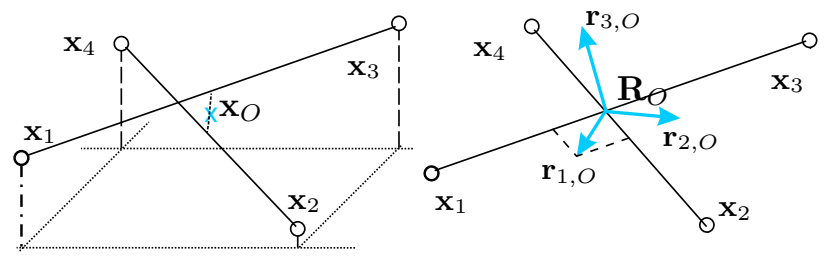

Fig. 2. Composition of the virtual object frame $\mathbf{H}_{O}=$ $\left[\mathbf{r}_{1, O}, \mathbf{r}_{2, O}, \mathbf{r}_{3, O}, \mathbf{x}_{O}\right]$ for $N=4$ fingers.

- The fingers are never singular during operation.

- The object geometry is convex.

\section{B. Object Frame}

To coordinate the finger motion, a virtual object is used similar to [15]. In this work however the object frame is defined uniquely by the positions of the fingertips. We propose a frame which is attached to the center of the fingertip positions

$$
\mathbf{x}_{O}(\boldsymbol{\theta})=\frac{\sum_{i=1}^{N} \mathbf{x}_{i}(\boldsymbol{\theta})}{N},
$$

where $\mathbf{x}_{i}(\boldsymbol{\theta}) \in \mathbb{R}^{3}$ represents the Cartesian fingertip position for finger $i$ w.r.t the base (frame 0). For simplicity we omit the dependence on $\boldsymbol{\theta}$ in the following. The orientation $\mathbf{R}_{O}=$ $\left[\mathbf{r}_{1, O}, \mathbf{r}_{2, O}, \mathbf{r}_{3, O}\right]$ is defined based on the Cartesian fingertip positions as well. For $N=4$ fingers the unitary vector $\mathbf{r}_{1, O}$ is defined in the plane spanned by the vectors being defined by the connections between fingers 1 and 3 and between fingers 2 and 4 , that is

$$
\tilde{\mathbf{r}}_{1, O}=\frac{\mathbf{x}_{1}-\mathbf{x}_{3}}{\left\|\mathbf{x}_{1}-\mathbf{x}_{3}\right\|}+\frac{\mathbf{x}_{2}-\mathbf{x}_{4}}{\left\|\mathbf{x}_{2}-\mathbf{x}_{4}\right\|}, \quad \mathbf{r}_{1, O}=\frac{\tilde{\mathbf{r}}_{1, O}}{\left\|\tilde{\mathbf{r}}_{1, O}\right\|} .
$$

The unitary vector $\mathbf{r}_{3, O}$ is defined perpendicular to this plane and $\mathbf{r}_{2, O}$ is defined such that $\mathbf{R}_{O} \in \mathrm{SO}(3)$ (compare Fig. 2):

$$
\begin{gathered}
\tilde{\mathbf{r}}_{3, O}=\widehat{\mathbf{x}_{1}-\mathbf{x}_{3}}\left(\mathbf{x}_{2}-\mathbf{x}_{4}\right), \quad \mathbf{r}_{3, O}=\frac{\tilde{\mathbf{r}}_{3, O}}{\left\|\tilde{\mathbf{r}}_{3, O}\right\|}, \\
\mathbf{r}_{2, O}=\widehat{\mathbf{r}_{3, O}} \mathbf{r}_{1, O},
\end{gathered}
$$

with the skew-symmetric operator $\widehat{\mathbf{w}}: \mathbb{R}^{3} \rightarrow \mathbb{R}^{3 \times 3}, \quad \widehat{\mathbf{w}}=$ $\left[\begin{array}{rrr}0, & -w_{3}, & w_{2} \\ w_{3}, & 0, & -w_{1} \\ -w_{2}, & w_{1}, & 0\end{array}\right]$. The virtual object frame $\mathbf{H}_{O}$ can be stacked to $\mathbf{H}_{O}=\left[\mathbf{R}_{O}, \mathbf{x}_{O}\right] \in \operatorname{SE}(3)$. Note that this representation has singularities if $\mathbf{x}_{j}-\mathbf{x}_{j+2}=0$ for $j=$ $1 \vee j=2$ or if $\left(\mathbf{x}_{1}-\mathbf{x}_{3}\right) \|\left(\mathbf{x}_{2}-\mathbf{x}_{4}\right)$. For common convex objects like boxes, cylinders or spheres these singularities pose no problem. If we want to extend to more than four fingers we can make sure that all elements of $\mathbf{H}_{O}$ are a function of each finger.

The virtual object frame is related to the real object frame if we assume that the relative contact points between the fingertips and the object do not change (neglecting rolling effects). 


\section{ObJect-Level Control LaW}

Our control law is based on

$$
\boldsymbol{\tau}=-\mathbf{D}(\boldsymbol{\theta}) \dot{\boldsymbol{\theta}}-\frac{\partial V_{d}}{\partial \boldsymbol{\theta}}(\boldsymbol{\theta})+\mathbf{g}(\boldsymbol{\theta}),
$$

with $\mathbf{D}(\boldsymbol{\theta})$ being a positive definite damping matrix. The desired potential

$$
V_{d}(\boldsymbol{\theta})=V_{O, t}(\boldsymbol{\theta})+V_{O, r}(\boldsymbol{\theta})+V_{C o n n}(\boldsymbol{\theta})
$$

is composed of the potentials to derive the translational and rotational object stiffness and the connecting stiffness, respectively (compare Fig. 3).

It is well known from the theory of passivity-based control that if we choose $V_{d}(\boldsymbol{\theta})$ to be positive semidefinite we obtain passivity for the closed-loop system. If $V_{d}(\boldsymbol{\theta})$ is positive definite stability of the closed-loop system can be concluded, and for asymptotic stability one can refer to LaSalle's theorem [17]. In the following each summand of $V_{d}(\boldsymbol{\theta})$ is designed to be positive semi-definite.

\section{A. Object-Level Stiffness}

The potentials related to the translational and the rotational object stiffness are chosen to be

$$
\begin{aligned}
& V_{O, t}(\boldsymbol{\theta})=\frac{1}{2}\left(\mathbf{x}_{O}-\mathbf{x}_{O, d e s}\right)^{T} \mathbf{R}_{O} \mathbf{K}_{O, t} \mathbf{R}_{O}^{T}\left(\mathbf{x}_{O}-\mathbf{x}_{O, d e s}\right) \\
& V_{O, r}(\boldsymbol{\theta})=2 \boldsymbol{\epsilon}_{b}^{T} \mathbf{K}_{O, r} \boldsymbol{\epsilon}_{b},
\end{aligned}
$$

where $\mathbf{x}_{O, d e s}, \boldsymbol{\epsilon}_{b}$ are the desired object position and the rotational error represented by the vector part of the unit quaternion [16], respectively. $\boldsymbol{\epsilon}_{b}$ is related to $\mathbf{R}_{O}^{T} \mathbf{R}_{O \text {,des }}$ with $\mathbf{R}_{O \text {,des }}$ specifying the desired object orientation. $\mathbf{K}_{O, t}, \mathbf{K}_{O, r} \in \mathbb{R}^{3 \times 3}$ represent the translational and rotational stiffness matrices represented in the object frame both chosen to be symmetric and positive definite.

To derive the rotational part of the control law we take the time derivative of $V_{O, r}(\boldsymbol{\theta})$ and use the differential mapping from angular object velocity relative to the base (frame 0 ) represented in body coordinates (frame $O$ ) $\boldsymbol{\omega}_{O, 0}$ to the rate of unit quaternions: $\dot{\boldsymbol{\epsilon}}_{d e s, b}=\mathbf{J}_{\boldsymbol{\omega} \boldsymbol{\epsilon}}(\boldsymbol{\theta}) \boldsymbol{\omega}_{O, 0}$. This yields to

$$
\dot{V}_{O, r}(\boldsymbol{\theta})=4 \boldsymbol{\epsilon}_{b}^{T} \mathbf{K}_{O, r} \mathbf{J}_{\boldsymbol{\omega} \epsilon}(\boldsymbol{\theta}) \boldsymbol{\omega}_{O, 0} .
$$

On the other hand together with

$$
\widehat{\omega_{O, 0}}=\mathbf{R}_{O}^{T} \dot{\mathbf{R}}_{O}
$$

we can write

$$
\boldsymbol{\omega}_{O, 0}=\left(\mathbf{R}_{O}^{T} \dot{\mathbf{R}}_{O}\right)^{\vee}=\left(\mathbf{R}_{O}^{T} \frac{\partial \mathbf{R}_{O}}{\partial \mathbf{x}^{T}} \frac{\partial \mathbf{x}}{\partial \boldsymbol{\theta}^{T}} \dot{\boldsymbol{\theta}}\right)^{\vee},
$$

where the operator $\mathbf{V}^{\vee}: \mathbb{R}^{3 \times 3} \rightarrow \mathbb{R}^{3}$, is defined as $\mathbf{V}^{\vee}=$ $\left[v_{32}, v_{13}, v_{21}\right]^{T}$ and $\mathbf{x}=\left[\mathbf{x}_{1}^{T}, \cdots, \mathbf{x}_{N}^{T}\right]^{T}$. Since expression (10) is linear in $\dot{\theta}$ we can re-parameterize this equation to obtain

$$
\boldsymbol{\omega}_{O, 0}=\mathbf{J}_{O, r}(\boldsymbol{\theta}) \dot{\boldsymbol{\theta}}
$$

In the following the dependence of the Jacobian relationships on $\boldsymbol{\theta}$ will be omitted. Inserting equation (11) in (8) and applying the inner product rule it follows that

$$
\dot{V}_{O, r}(\boldsymbol{\theta})=\dot{\boldsymbol{\theta}}^{T} \underbrace{\mathbf{J}_{O, r}^{T} \overbrace{4 \mathbf{J}_{\omega \epsilon}^{T} \mathbf{K}_{O, r} \boldsymbol{\epsilon}_{b}}^{\mathbf{m}_{O}}}_{\frac{\partial V_{O, r}(\boldsymbol{\theta})}{\partial \boldsymbol{\theta}}} .
$$

The derivation of the translational part starts similarly by considering the time derivative of the potential function

$$
\dot{V}_{O, t}(\boldsymbol{\theta})=\left(\mathbf{x}_{O}-\mathbf{x}_{O, d e s}\right)^{T} \mathbf{R}_{O} \mathbf{K}_{O, t} \frac{d}{d t}\left\{\mathbf{R}_{O}^{T}\left(\mathbf{x}_{O}-\mathbf{x}_{O, d e s}\right)\right\} .
$$

Looking more precisely at the time derivative in the equation above which we can transform to

$$
\begin{aligned}
& \frac{d}{d t}\left\{\mathbf{R}_{O}^{T}\left(\mathbf{x}_{O}-\mathbf{x}_{O, d e s}\right)\right\}= \\
& \dot{\mathbf{R}}_{O}^{T}\left(\mathbf{x}_{O}-\mathbf{x}_{O, d e s}\right)+\mathbf{R}_{O}^{T} \frac{\partial \mathbf{x}_{O}}{\partial \mathbf{x}^{T}} \frac{\partial \mathbf{x}}{\partial \boldsymbol{\theta}^{T}} \dot{\boldsymbol{\theta}}= \\
& {\left[\left(\mathbf{R}_{O}^{T}\left(\mathbf{x}_{O}-\mathbf{x}_{O, d e s}\right)\right)^{\tilde{S}}\left\{\mathbf{J}_{O, r}\right\}_{O}+\mathbf{R}_{O}^{T} \frac{\partial \mathbf{x}_{O}}{\partial \mathbf{x}^{T}} \frac{\partial \mathbf{x}}{\partial \boldsymbol{\theta}^{T}}\right] \dot{\boldsymbol{\theta}}}
\end{aligned}
$$

using equations (9), (11) and the skew-symmetric identities $\hat{\mathbf{v}}^{T}=-\hat{\mathbf{v}}$ and $\hat{\mathbf{v}} \mathbf{w}=-\hat{\mathbf{w}} \mathbf{v}$ in the last step. By inserting equation (14) in (13) and evaluating $\frac{\partial \mathbf{x}_{O}^{T}}{\partial \mathbf{x}}=\frac{1}{N} \mathrm{I}_{3 \mathrm{~N} \times 3}$ and recognizing the hand Jacobian represented in the base frame $\left\{\mathbf{J}_{H}\right\}_{0}=\frac{\partial \mathbf{x}}{\partial \boldsymbol{\theta}^{T}}$ we obtain

$$
\dot{V}_{O, t}(\boldsymbol{\theta})=\dot{\boldsymbol{\theta}}^{T} \underbrace{\mathbf{J}_{O, t}^{T} \overbrace{\mathbf{K}_{O, t} \mathbf{R}_{O}^{T}\left(\mathbf{x}_{O}-\mathbf{x}_{O, d e s}\right)}^{\mathbf{f}_{O}}}_{\frac{\partial V_{O, t}(\boldsymbol{\theta})}{\partial \boldsymbol{\theta}}},
$$

with

$$
\mathbf{J}_{O, t}=\left[\frac{1}{N}\left\{\mathbf{J}_{H}\right\}_{0}^{T} \mathrm{I}_{3 \mathrm{~N} \times 3} \mathbf{R}_{O}-\mathbf{J}_{O, r}^{T}\left(\mathbf{R}_{O}^{T}\left(\mathbf{x}_{O}-\mathbf{x}_{O, d e s}\right)\right)^{T}\right]^{T} .
$$

The gradients of the potentials related to the rotational and translational stiffness $\frac{\partial V_{O, r}(\boldsymbol{\theta})}{\partial \boldsymbol{\theta}}, \frac{\partial V_{O, t}(\boldsymbol{\theta})}{\partial \boldsymbol{\theta}}$ (eq. (12),(15)) contribute to the control law (5).

\section{B. Connecting Stiffness}

Up to now the $6 \mathrm{D}$ spring related to object motion is derived. The following part addresses the springs connecting the fingertips with the virtual object frame. With these springs we can adjust the grasping forces. The setpoints $l_{i, d e s}$ for the stiffness relationship as defined below are chosen manually to suffice the frictional constraints. The contact model is assumed to be PCWF and considering the $3 \mathrm{DOF}$ of each finger we propose to use 1D springs connecting each fingertip position $\mathbf{x}_{i}$ with the virtual object frame (compare Fig. 3). The potential is chosen to be spherical for each fingertip:

$$
V_{\text {Conn }}(\boldsymbol{\theta})=\frac{1}{2} \sum_{i=1}^{N} K_{C o n n, i}\left[\left\|\boldsymbol{\Delta} \mathbf{x}_{i}\right\|-l_{i, d e s}\right]^{2}
$$

with $\Delta \mathbf{x}_{i}=\mathbf{x}_{i}-\mathbf{x}_{O}$ and $l_{i, \text { des }}$ as the desired distance from the fingertip $i$ to the virtual object frame $\mathbf{x}_{O}$, and $K_{C o n n, i}>0$ the corresponding connecting stiffness.

The contribution to the control law (5) can be obtained and simplified to

$$
\frac{\partial V_{C o n n}(\boldsymbol{\theta})}{\partial \boldsymbol{\theta}}=\left\{\mathbf{J}_{H}\right\}_{0}^{T} \sum_{i=1}^{N}\left[\frac{\partial \boldsymbol{\Delta} \mathbf{x}_{i}^{T}}{\partial \mathbf{x}} \frac{K_{C o n n, i}\left(\left\|\boldsymbol{\Delta} \mathbf{x}_{i}\right\|-l_{i, d e s}\right)}{\left\|\boldsymbol{\Delta} \mathbf{x}_{i}\right\|} \boldsymbol{\Delta} \mathbf{x}_{i}\right],
$$




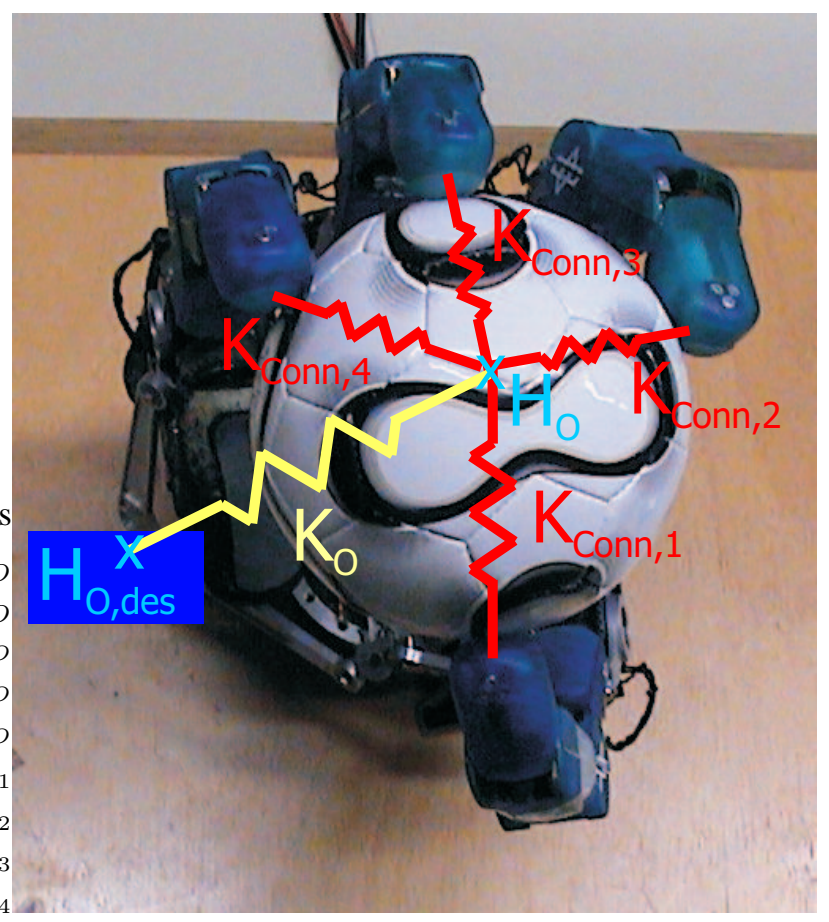

Fig. 3. DLR Hand II grasping a toy ball with a diameter of $0.11 \mathrm{~m}$ superimposed by the virtual springs defined by the potential functions in equation (6).

with

$$
\frac{\partial \boldsymbol{\Delta} \mathbf{x}_{i}^{T}}{\partial \mathbf{x}}=\left[\begin{array}{ccccccc}
a \mathrm{I}_{3 \times 3} & \cdots & a \mathrm{I}_{3 \times 3} & b \mathrm{I}_{3 \times 3} & a \mathrm{I}_{3 \times 3} & \cdots & a \mathrm{I}_{3 \times 3} \\
1 & & i-1 & i & i+1 & & N
\end{array}\right.
$$

and $a=\left(1-\frac{1}{N}\right), b=-\frac{1}{N}$.

Rewriting this equation in matrix form gives further simplification

$$
\frac{\partial V_{C o n n}(\boldsymbol{\theta})}{\partial \boldsymbol{\theta}}=\mathbf{J}_{\text {conn }}^{T} \underbrace{\mathbf{K}_{\text {Conn }}\left[\begin{array}{c}
\left\|\mathbf{\Delta x}_{1}\right\|-l_{1, \text { des }} \\
\vdots \\
\left\|\mathbf{x}_{N}\right\|-l_{N, \text { des }}
\end{array}\right]}_{\mathbf{f}_{\text {Conn }}},
$$

with

$$
\mathbf{J}_{\text {conn }}^{T}=\left\{\mathbf{J}_{H}\right\}_{0}^{T}\left[\begin{array}{llll}
\frac{\partial \boldsymbol{\Delta} \mathbf{x}_{1}^{T}}{\partial \mathbf{x}} \frac{\boldsymbol{\Delta} \mathbf{x}_{1}}{\left\|\boldsymbol{\Delta} \mathbf{x}_{1}\right\|} & \cdots & \frac{\partial \boldsymbol{\Delta} \mathbf{x}_{N}^{T}}{\partial \mathbf{x}} \frac{\boldsymbol{\Delta} \mathbf{x}_{N}}{\left\|\boldsymbol{\Delta} \mathbf{x}_{N}\right\|}
\end{array}\right]
$$

We use the concept of virtual work to recognize $\|\Delta \dot{\mathbf{x}}\|=\left[\left\|\Delta \dot{\mathbf{x}}_{1}\right\|, \ldots,\left\|\Delta \dot{\mathbf{x}}_{N}\right\|\right]^{T}=\mathbf{J}_{\text {conn }} \dot{\boldsymbol{\theta}}$ as a differential relationship between the distance of each finger to the virtual object and the hand configuration. $\mathbf{K}_{C o n n}=$ $\operatorname{diag}\left\{K_{C o n n, 1}, \ldots, K_{C o n n, N}\right\}$ is the stiffness matrix of the connecting springs. ${ }^{1}$

In equations (11), (16) and (21) we obtained the differential mapping from the hand motion to object motions and change

\footnotetext{
${ }^{1}$ Throughout this paper $\operatorname{diag}\{\cdot\}$ applied to a matrix extracts its diagonal elements. Applied to a vector the output is a matrix having the vector on its diagonal.
}

of distance between the fingertips to the object:

$$
\underbrace{\left[\begin{array}{c}
\dot{\mathbf{x}}_{O} \\
\boldsymbol{\omega}_{O, 0} \\
\|\boldsymbol{\Delta} \dot{\mathbf{x}}\|
\end{array}\right]}_{\dot{\mathbf{x}}}=\underbrace{\left[\begin{array}{c}
\mathbf{J}_{O, t} \\
\mathbf{J}_{O, r} \\
\mathbf{J}_{c o n n}
\end{array}\right]}_{\mathbf{J}_{t o t}} \dot{\boldsymbol{\theta}}
$$

where $\dot{\overline{\mathbf{x}}} \in \mathbb{R}^{(6+N)}$ represents generalized coordinates. This stacked Jacobian is useful for a compact derivation of the damping parameters.

The object workspace can be a function of

- the hand joint limits,

- the manipulated object geometry,

- and the admissible regions of contact on the fingertips.

If the object workspace is known, we can add another potential to equation (6) to generate a repulsive force close to the objectlevel workspace boundary.

\section{DAMPING DESIGN}

So far, only the stiffness behavior has been derived. Adding damping terms we can shape the transient behavior based on the desired stiffness and the inertial properties of the handobject system. Let $\mathbf{D}_{\overline{\mathbf{x}}}$ be the damping matrix related to the generalized coordinates $\overline{\mathbf{x}}$ as defined in equation (22) we write

$$
\mathbf{D}(\boldsymbol{\theta})=\mathbf{J}_{t o t}^{T} \mathbf{D}_{\overline{\mathbf{x}}}(\boldsymbol{\theta}) \mathbf{J}_{t o t} .
$$

In order to assign the desired behavior to the closed-loop system we use the corresponding stiffness matrix $\mathbf{K}_{\overline{\mathbf{x}}}=$ blockdiag $\left\{\mathbf{K}_{O, t}, \mathbf{K}_{O, r}, \mathbf{K}_{C o n n}\right\}$, the joint-level inertia matrix of the hand $\mathbf{M}(\boldsymbol{\theta})$ and the inertia matrix of the object $\mathbf{M}_{O} \in$ $\mathbb{R}^{6 \times 6}$.

The kinetic energy of the hand-object system can be written as

$$
\mathcal{T}=\frac{1}{2} \dot{\boldsymbol{\theta}}^{T} \mathbf{M}(\boldsymbol{\theta}) \dot{\boldsymbol{\theta}}+\frac{1}{2}\left[\dot{\mathbf{x}}_{O}^{T}, \boldsymbol{\omega}_{O, 0}^{T}\right] \mathbf{M}_{O}\left[\begin{array}{c}
\dot{\mathbf{x}}_{O} \\
\boldsymbol{\omega}_{O, 0}
\end{array}\right]
$$

Mapping the joint-level inertia matrix to generalized coordinates gives $\mathbf{M}_{H, \bar{x}}(\boldsymbol{\theta})=\left(\mathbf{J}_{t o t} \mathbf{M}(\boldsymbol{\theta})^{-1} \mathbf{J}_{t o t}^{T}\right)^{-1}$. Extending the second summand in (24) to generalized coordinates and factorizing $\dot{\overline{\mathbf{x}}}$ out, we can identify an inertia matrix related to generalized coordinates as

$$
\mathbf{M}_{\bar{x}}(\boldsymbol{\theta})=\mathbf{M}_{H, \bar{x}}(\boldsymbol{\theta})+\left[\begin{array}{c}
\mathrm{I}_{6 \times 6} \\
0_{N \times 6}
\end{array}\right] \mathbf{M}_{O}\left[\mathrm{I}_{6 \times 6}, 0_{6 \times N}\right] .
$$

In our particular case the diagonal elements within the inertia matrix $\mathbf{M}_{\bar{x}}(\boldsymbol{\theta})$ are dominating, so that only those values are considered for the damping design. With this consideration we do not need to calculate the complete $\mathbf{M}_{\bar{x}}(\boldsymbol{\theta})$, but we can look at $\left(\operatorname{diag}\left\{\operatorname{diag}\left\{\left(\mathbf{J}_{\text {tot }} \mathbf{M}(\boldsymbol{\theta})^{-1} \mathbf{J}_{\text {tot }}^{T}\right)\right\}\right\}\right)^{-1}$ avoiding the inversion of a dense $(6+N) \times(6+N)$ matrix. The diagonal elements of $\mathbf{D}_{\bar{x}}(\boldsymbol{\theta})$ are chosen as

$$
\mathbf{D}_{\bar{x}, i i}(\boldsymbol{\theta})=2 \xi_{i} \sqrt{\mathbf{M}_{\bar{x}, i i}(\boldsymbol{\theta}) \mathbf{K}_{\bar{x}, i i}}
$$

where $\xi_{i} \in[0,1]$ represents the desired damping factor corresponding to the coordinate $\bar{x}_{i}$. For each generalized coordinate the damping is limited to a maximum value. 

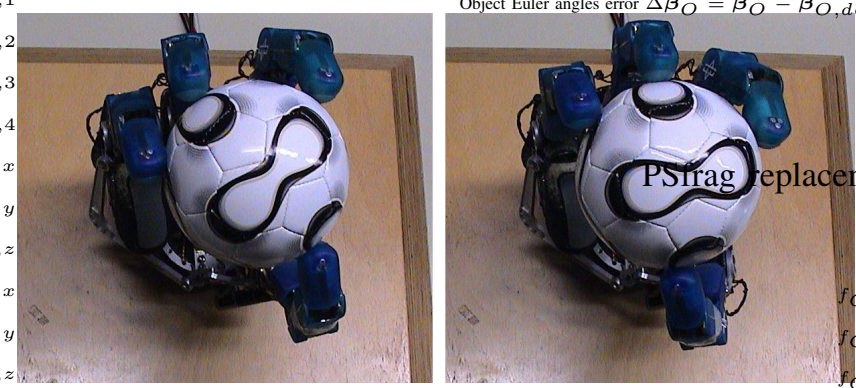

Fig. 4. Hand holding the ball before (left) and after the rotation around the object $z$-axis with $\Delta \beta_{O, z}=0.6 \mathrm{rad}$ (right).

If we want to use the complete inertia matrix we can apply the damping design by double diagonalization presented in [18].

Since the damping matrix $\mathbf{D}(\boldsymbol{\theta})$ is determined for the case including the object inertia we provide a conservative choice of damping in the case the object is not yet grasped. In case a finger looses contact during manipulation its inertia is less than in contact with the object. Leaving the damping coefficient constant the effective damping for this finger will be increased enforcing stability.

\section{EXPERIMENTS}

The presented control law is implemented on the DLR Hand II. The hand has four fingers with 3 DOF each, resulting in overall 12 DOF. For each joint motor- and link-side position and link-side torque are measured. In the distal link a 6 DOF force-/torque sensor is integrated covered by a hemispherical fingertip. Details on the hand hardware can be found in [19]. The control law was implemented using the Real-time Workshop of MATLAB/SIMULINK in combination with the tool chain offered by RT-LAB [20]. The code generated from the SIMULINK model runs on QNX on a Pentium IV with $3 \mathrm{GHz}$ with a controller sample time of $1 \mathrm{~ms}$.

The desired control torque from equation (5) is used as set point for a low-level torque controller which is based on the joint torque measurement, and offline estimated static and viscous motor friction parameters.

The control law is evaluated in two experiments: A) Pure object rotation and B) Pure object translation. For both experiments a toy ball with a diameter of $0.11 \mathrm{~m}$ and a weight of $0.1 \mathrm{~kg}$ is grasped.

The stiffness matrices and the overall damping coefficient $\xi$ are parameterized as described in Table I.

\begin{tabular}{|c|c|c|c|}
\hline $\operatorname{diag}\left\{\mathbf{K}_{O, t}\right\}\left[\frac{N}{m}\right]$ & $\operatorname{diag}\left\{\mathbf{K}_{O, r}\right\}\left[\frac{N m}{\text { rad }}\right]$ & $\operatorname{diag}\left\{\mathbf{K}_{\text {Conn }}\right\}\left[\frac{N}{m}\right]$ & $\xi$ \\
\hline$[2000,2000,2000]$ & {$[10,10,10]$} & {$[150,150,150,150]$} & 0.7 \\
\hline \multicolumn{4}{|c|}{ TABLE I }
\end{tabular}

CONTROLLER PARAMETRIZATION.

\section{A. Object Rotation}

The grasped ball is rotated around the virtual object $z$-axis by commanding a step $\Delta \beta_{O, z}=0.6 \mathrm{rad}$ (see Fig. 4). The step response of the object impedance torque $\mathbf{m}_{O}$ starting

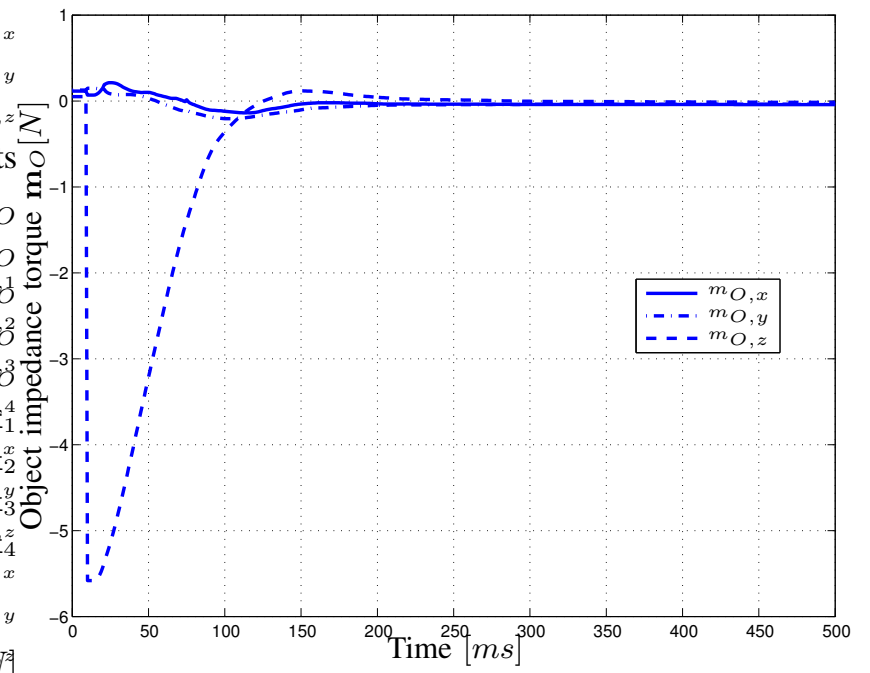

Fig. 5. Object impedance torque $\mathbf{m}_{O}$ during the rotation around the object $z$-axis with $\Delta \beta_{O, z}=0.6 \mathrm{rad}$.

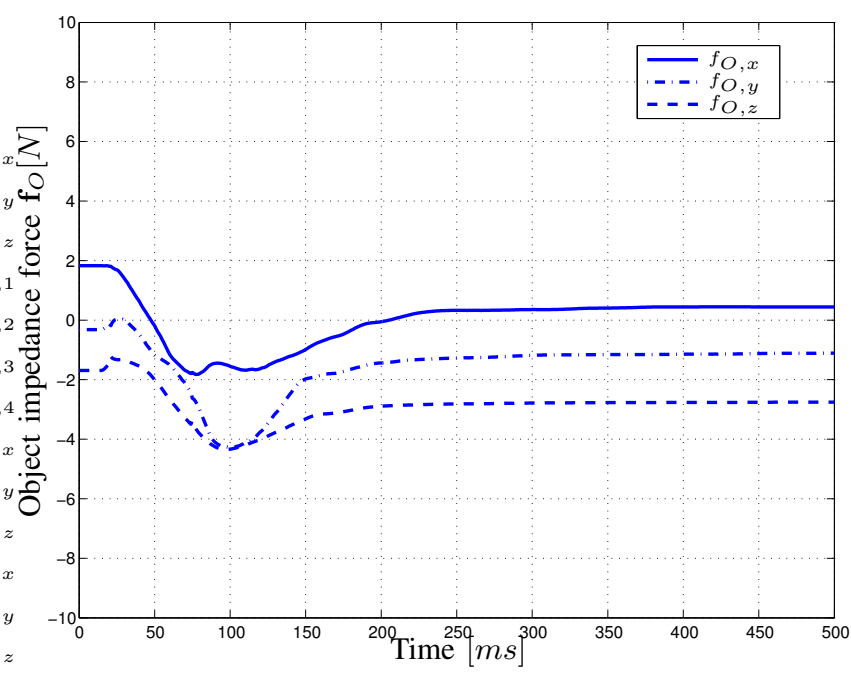

Fig. 6. Object impedance force $\mathbf{f}_{O}$ during the rotation around the object $z$-axis with $\Delta \beta_{O, z}=0.6 \mathrm{rad}$.

at $t=10 \mathrm{~ms}$ is presented in Fig. 5, where a high peak in the $z$-component can be observed. In steady-state the overall impedance torque converges to a value smaller than $0.06 \mathrm{Nm}$. The residual error stems from joint friction ${ }^{2}$. Since the fingers are interconnected over the object, the remaining friction effects sum up over all fingers. In Fig. 6 we observe changes of the object impedance force $f_{O}$ in the magnitude of $2 \mathrm{~N}$ during the rotation indicating that the coupling between rotation and translation is small. Looking at the connecting forces $\mathbf{f}_{\text {Conn }}$ in Fig. 7, two remarks can be stated: Firstly, the variations of the connecting forces are about half the magnitude of the changes of the object impedance force. Secondly, relating $f_{C o n n, 1}$ to the thumb and considering its opposing position relative to the other fingers (compare Fig. 3),

\footnotetext{
${ }^{2}$ The friction compensation in the torque controller is chosen rather conservative to ensure stability.
} 


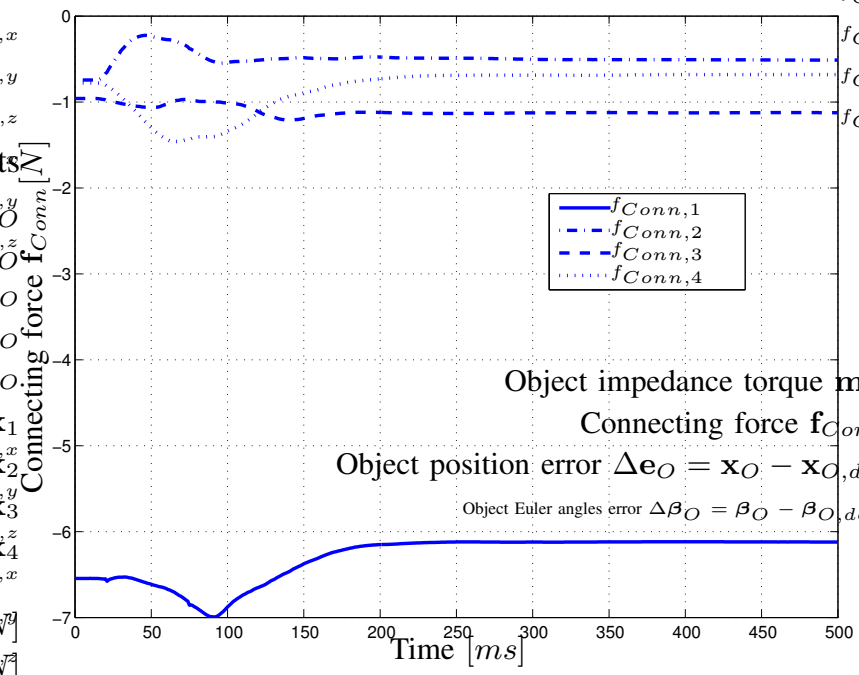

Fig. 7. Connecting forces $\mathbf{f}_{\text {Conn }}$ during the rotation around the object $z$-axis with $\Delta \beta_{O, z}=0.6 \mathrm{rad}$.

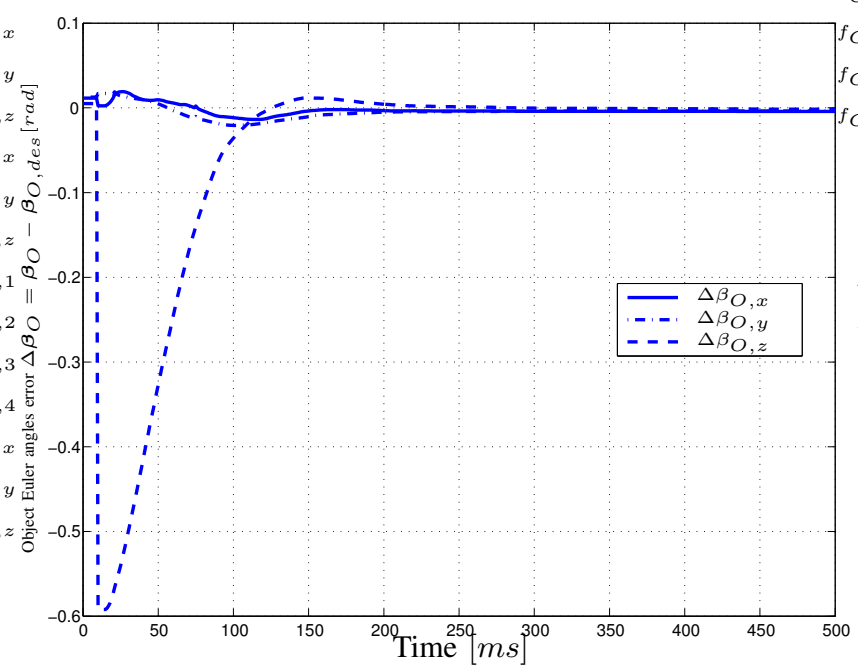

Fig. 8. Object Euler angles $\beta_{O}$ during the rotation around the object $z$-axis with $\Delta \beta_{O, z}=0.6 \mathrm{rad}$.

one can see that the thumb has to work against the opposing fingers in this hand configuration. The rotational object error depicted in Fig. 8 has a very similar transient behavior as the corresponding object impedance torque $\mathbf{m}_{O}$.

\section{B. Object Translation}

In the second experiment, we commanded a step in the translation of the object along the $y$-axis with $\Delta x_{O, y}=$ $0.06 \mathrm{~m}$ (Fig. 9). In Fig. 10 a jump of $\mathbf{f}_{O}$ at $t=10 \mathrm{~ms}$ can be observed. At steady-state $\mathbf{f}_{O}$ converges to $4.6 \mathrm{~N}$. $\mathbf{m}_{O}$ is influenced by this motion in the magnitude of $0.5 \mathrm{Nm}$ showing a small coupling effect between object translation and rotation. Again, in Fig. 12 it can be observed the unbalance of $\mathbf{f}_{\text {Conn }}$ for the same reason as in the previous experiment. Note that the closed-loop eigenfrequencies for the rotational and the translational stiffness have the same magnitude. The

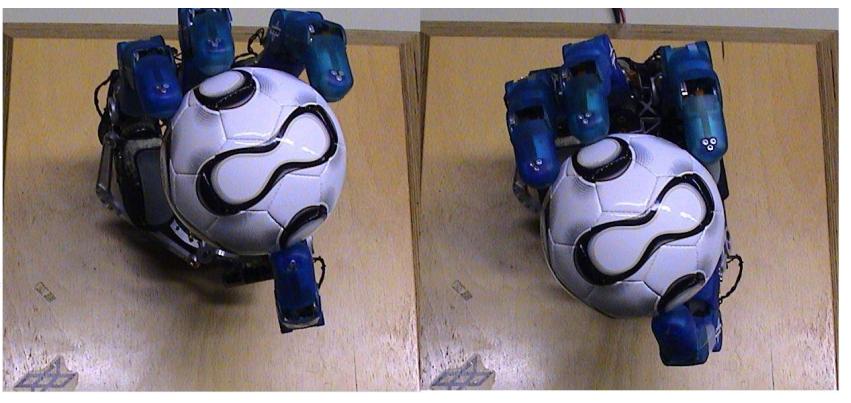

Fig. 9. Hand holding the ball before (left) and after the translating the object along the $y$-axis with $\Delta x_{O, y}=0.06 \mathrm{~m}$ (right).

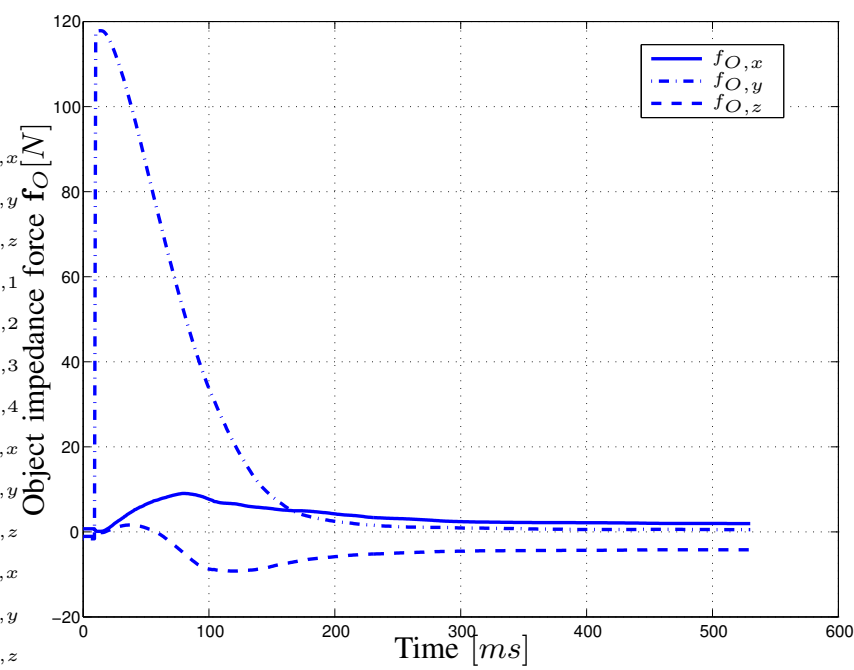

Fig. 10. Object impedance force $\mathbf{f}_{O}$ during translating the object along the $y$-axis with $\Delta x_{O, y}=0.06 \mathrm{~m}$.

error in object position behaves similar to $\mathbf{f}_{O}$ and is therefore not shown.

\section{CONCLUSIONS AND FUture WORK}

A robust and intuitive control law for object-level manipulation was presented. Given an initial position for the fingers we can specify object translations, rotations, and connecting forces. The basis for this control was the virtual object frame which is defined by the positions of the fingertips. Realizing the object motion and grasp force control as spring-damper systems we need not to track the contact states. Stability of the control was ensured even if a finger slips or lifts off the object. The controller was implemented on the DLR Hand II and experimental results confirmed its performance. Clearly, the evaluation of the friction constraints is important and will be integrated in future work. The planning of contact points on arbitrary objects can be applied (e. g. see [21]). The connecting forces, respectively how to set $l_{i, d e s}$, will be included. Furthermore, the design of the connecting springs will be investigated since they are a simple model to represent soft fingertips.

The proposed control law has a similar structure to the robot arm controller for the DLR light-weight robots proposed 


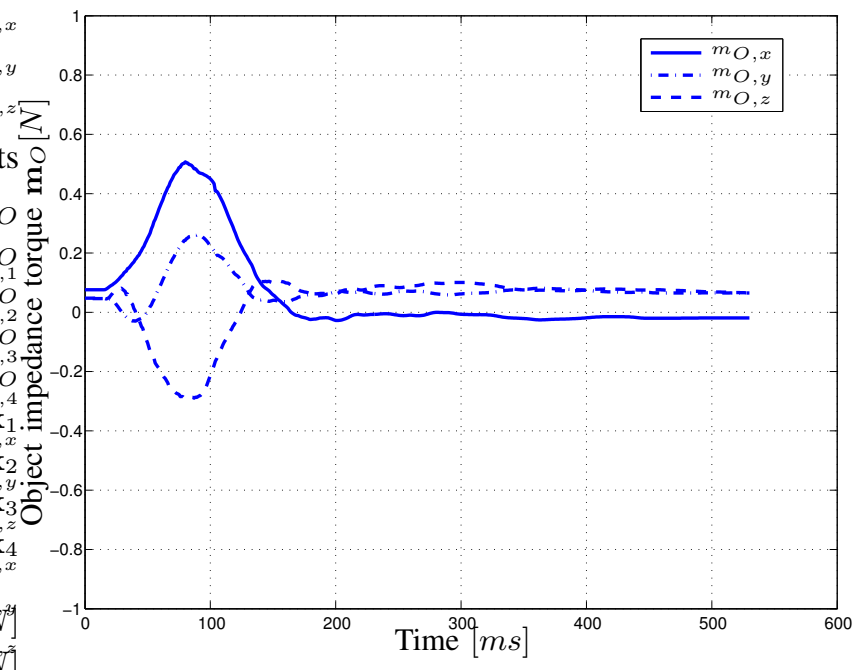

Fig. 11. Object impedance torque $\mathbf{m}_{O}$ during translating the object along the $y$-axis with $\Delta x_{O, y}=0.06 \mathrm{~m}$.

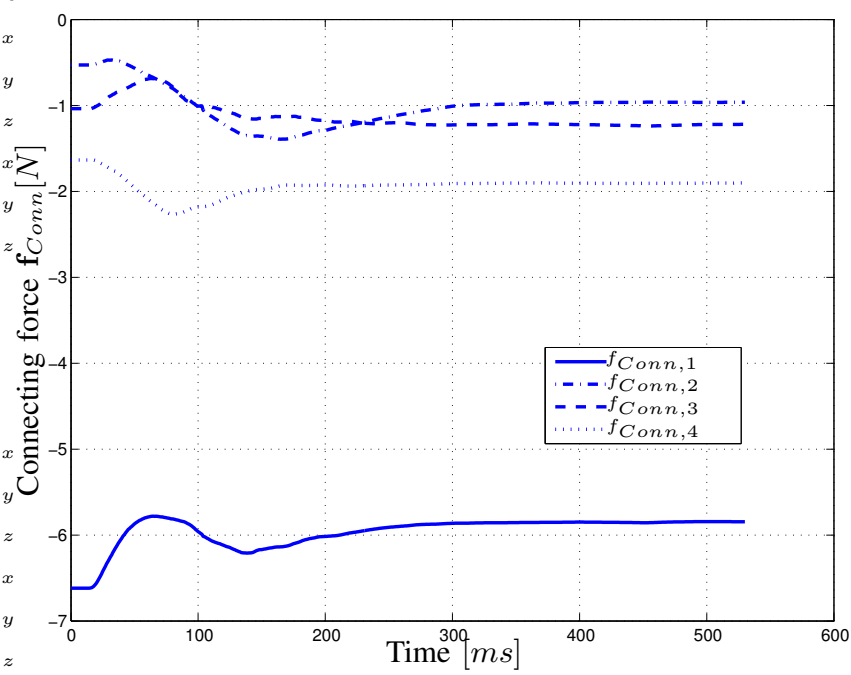

Fig. 12. Connecting forces $\mathbf{f}_{\text {Conn }}$ during translating the object along the $y$-axis with $\Delta x_{O, y}=0.06 \mathrm{~m}$.

in [18]. The DLR Hand II is mounted on the end-effector presenting a hand-arm system with $7+12$ DOF. We can now extend the presented object-level control law on the whole object-hand-arm system.

\section{REFERENCES}

[1] A. Bicchi, Hand for Dexterous Manipulation and Robust Grasping: A Difficult Road Towards Simplicity, IEEE Transactions on Robotics and Automation, Vol. 16, No. 6, Dec. 2000, pp. 652-662.

[2] Ch. Borst, M. Fischer, S. Haidacher, H. Liu, G. Hirzinger, DLR Hand II: Experiments and Experiences with an Anthropomophic Hand, IEEE International Conference on Robotics and Automation, Taipei, Taiwan, 2003, pp. 684-689.
[3] L. Biagiotti, H. Liu, G. Hirzinger, C. Melchiorri, Cartesian Impedance Control for Dexterous Manipulation, IEEE/RSJ International Conference on Intelligent Robots and Systems, Las Vegas, USA, 2003, pp. 32703275.

[4] S. A. Schneider and R. H. Cannon, Jr., Object Impedance Control for Cooperative Manipulation: Theory and Experimental Results, IEEE Transactions on Robotics and Automation, Vol. 8, No. 3, Jun. 1992, pp. 383-394.

[5] S. Arimoto, J.-H. Bae, and K. Tahara, Stability on a Manifold: Simultaneous Realization of Grasp and Orientation Control of an Object by a Pair of Robot Fingers, IEEE International Conference on Robotics and Automation, Taipei, Taiwan, 2003, pp. 2336-2343.

[6] D. Williams, and O. Khatib, The virtual linkage: A model for internal forces in multi-grasp manipulation, IEEE International Conference on Robotics and Automation, Atlanta, USA, pp. 1025-1030, 1993.

[7] O. Khatib, Inertial Properties in Robotics Manipulation: An Object-Level Framework, International Journal of Robotics Research, vol. 14, no. 1, February 1995, pp. 19-36.

[8] F. Caccavale, P. Chiacchio, and S. Chiaverini, Stability Analysis of a Joint Space Control Law for a Two-Manipulator System, IEEE Transactions on Automatic Control, Vol. 44, No. 1, pp. 85-88, 1999.

[9] I. D. Walker, R. A. Freeman, and S. I. Marcus, Analysis of motion and internal loading of objects grasped by multiple cooperating manipulators, International Journal of Robotics Research, Vol. 10, No. 4, 1991, pp. 396-409.

[10] T. Naniwa and K. Wada, Experimental study of coordinated control of multifingered hands with the kinetostatic filtering method, Advanced Robotics, Vol. 19, No. 2, pp. 383-394, 2005.

[11] Xiaoping Yun and V. R. Kumar, An Approach to Simultaneous Control of Trajectory and Interaction Forces in Dual-Arm Configurations, IEEE Transactions on Robotics and Automation, Vol. 7, No. 5, Oct. 1991, pp. 618-624.

[12] J. T. Wen, and K. Kreutz-Delgado, Motion and Force Control of Multiple Robotic Manipulators, Automatica, Vol. 28, No. 4, 1992, pp. 729-743, 1993.

[13] R. M. Murray, Z. Li, and S. S. Sastry, A Mathematical Introduction to Robotic Manipulation, Boca Raton: CRC Press, 1994.

[14] R. Bonitz and T. Hsia, Internal Force-based Impedance Control for Cooperating Manipulators, IEEE Transactions on Robotics and Automation, Vol. 12, No. 1, Feb. 1996, pp. 78-89.

[15] S. Stramigioli, Modeling and IPC Control of Interactive Mechanical Systems: A Coordiante-free Approach, Springer Verlag, 2001.

[16] C. Natale, Interaction Control of Robot Manipulators: Six-Degrees-ofFreedom Tasks, Springer Verlag, 2003.

[17] W. Khalil and E. Dombre Modeling, Identification \& Control of Robots, Hermes Penton Science, 2002.

[18] A. Albu-Schäffer, C. Ott, G. Hirzinger, A Passivity Based Cartesian Impedance Controller for Flexible Joint Robots - Part II: Full State Feedback, Impedance Design and Experiments, IEEE International Conference on Robotics and Automation, New Orleans, USA, 2004, pp. 2666-2672.

[19] J. Butterfaß, M. Grebenstein, H. Liu, G. Hirzinger, DLR-Hand II: Next Generation of a Dextrous Robot Hand, IEEE International Conference on Robotics and Automation, Seoul, Korea, 2001, pp. 109-114.

[20] B. Bäuml and G. Hirzinger, Agile Robot Development (aRD): A Pragmatic Approach to Robotic Software, accepted at IEEE/RSJ International Conference on Intelligent Robots and Systems, Beijing, China, 2006.

[21] Ch. Borst, M. Fischer, G. Hirzinger, Grasping the Dice by Dicing the Grasp, IEEE/RSJ International Conference on Intelligent Robots and Systems, Las Vegas, USA, 2003, pp. 3692-3697. 